\title{
An efficient method for synthesising unsymmetrical silaketals: substrates for ring-closing, including macrocycle-closing, metathesis $\uparrow$
}

\author{
Christopher Cordier, ${ }^{a, b}$ Daniel Morton, ${ }^{a, b}$ Stuart Leach, ${ }^{a, b}$ Thomas Woodhall, ${ }^{a, b}$ Catherine O'Leary-Steele, ${ }^{a, b}$ \\ Stuart Warriner ${ }^{a, b}$ and Adam Nelson ${ }^{* a, b}$
}

Received 19th March 2008, Accepted 20th March 2008

First published as an Advance Article on the web 10th April 2008

DOI: $10.1039 / b 804769 n$

Diisopropylsilyl ethers were activated with $N$-bromosuccinimide, and reacted with a fluorous-tagged alcohol, to yield tethered substrates for ring-closing metathesis reactions.

The use of temporary tethers between reactants is an extremely valuable approach in synthetic organic chemistry. ${ }^{1}$ The approach can promote selective reaction between the tethered components, often under much milder conditions than the corresponding intermolecular process. Furthermore, reactions between the tethered components often proceed with stereoselectivities that complement, or improve on, those observed in intermolecular reactions.

Silicon tethers can control the cross-metathesis of pairs of allylic or homoallylic alcohols (e.g. 1 and 2, Scheme 1). After tethering of the reactants $($ e.g. $\rightarrow \mathbf{3})$, a ring-closing metathesis reaction gives a cyclic silaketal; silaketal cleavage $(e . g .4 \rightarrow 5)$ then yields the $Z$ isomer of net cross-metathesis of the components. The approach has been used in target ${ }^{2}$ and library ${ }^{3}$ synthesis, for example in the synthesis of attenol $\mathrm{A},{ }^{2 a}$ mucocin, ${ }^{2 b}$ and the epothilones. ${ }^{2 c}$ Furthermore, tethering metathesis reactions can lead to longrange diastereoselection. ${ }^{4}$

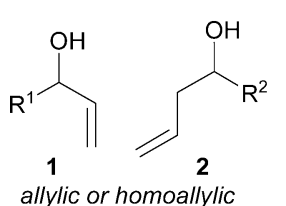

alcohols

unsymmetrical
silaketal formation<smiles>[R]C(C=C)O[SiH]([R])OC([R])CC=C</smiles>

3
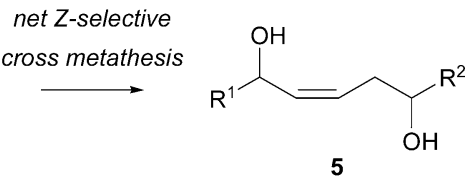

5
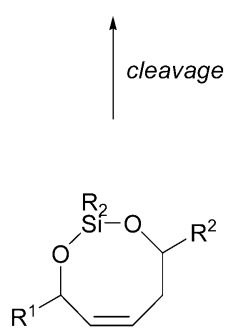

4
Scheme 1 Net $Z$-selective cross-metathesis of an allylic and a homoallylic alcohol.

${ }^{a}$ School of Chemistry, University of Leeds, Leeds, UK, LS2 9JT. E-mail: a.s.nelson@leeds.ac.uk; Fax: +44 (0)113 343 6565; Tel: +44 (0)1133436502

${ }^{b}$ Astbury Centre for Structural Molecular Biology, University of Leeds, Leeds, UK LS2 9JT

$\dagger$ Electronic supplementary information (ESI) available: Full experimental details and copies of NMR spectra for key compounds. See DOI: $10.1039 / \mathrm{b} 804769 \mathrm{n}$
A number of methods ${ }^{2,4,5}$ have been developed for the selective synthesis of the required unsymmetrical silaketals. Usually, one of the alcohols is reacted with an excess of a volatile dichlorosilane. ${ }^{2 a-b, 4}$ Alternatively, reaction of the anion of one of the alcohols with a dichlorosilane can give the monosubstituted reagent needed to prepare selectively the unsymmetrical product. ${ }^{2 c}$ In this communication, we describe an improved efficient and selective method for preparing unsymmetrical silaketal substrates for ring-closing (including macrocycle-closing) metathesis reactions.

We required an efficient method for the formation of unsymmetrical silaketals from the fluorous-tagged alcohol ${ }^{6} \mathbf{6}$ (Scheme 2). The key design features of the fluorous-tagged linker $\mathbf{6}$ are summarised in Scheme 3. A fluorous tag was incorporated to facilitate the purification by fluorous-solid phase extraction ${ }^{7}$ (F-SPE) of the required silaketals 7 from excess reagents used. The linker 6 was designed ${ }^{6}$ such that metathesis of the unsymmetrical silaketals (e.g. 7d) would release the required cyclic products (e.g. 9) from the fluorous tag, thus facilitating purification by F-SPE. Subsequent cyclisation of $\mathbf{1 0}(\rightarrow \mathbf{1 1})$ would then regenerate the catalytically active methylene complex. ${ }^{8}$
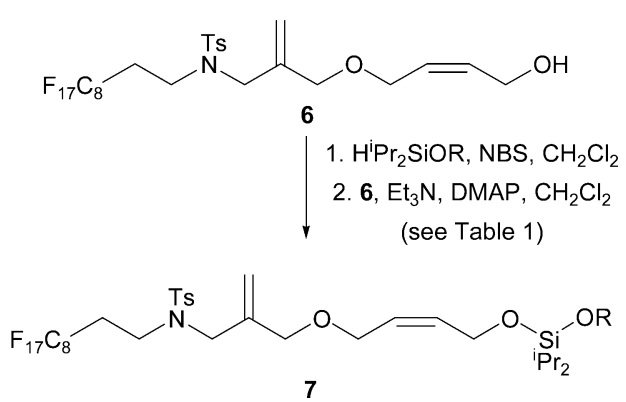

Scheme 2 Synthesis of fluorous-tagged unsymmetrical silaketals $\mathbf{7 a - 1}$. See Fig. 1 for definitions of the R groups.

To start with, we investigated Malacria's method for the formation and activation of diisopropylsilyl ethers (entry 1, Table 1). ${ }^{9}$ Hence the alcohol 12 was silylated, activated with NBS, and the resulting bromosilane reacted with the fluorous-tagged alcohol $\mathbf{6}$. Unfortunately, only a trace of the required silaketal was isolated, and the fluorous-tagged alcohol was recovered in $>98 \%$ yield.

We therefore re-investigated the activation of the racemic diisopropylsilyl ether rac-13d with NBS. NBS was added to a sample of the silyl ether $\mathbf{1 3 d}$ in $\mathrm{CDCl}_{3}$, and the reaction was monitored by $500 \mathrm{MHz}{ }^{1} \mathrm{H}$ NMR spectroscopy (see Fig. 2). Within $15 \mathrm{~min}$, the silyl ether 13d had been consumed, and had been converted cleanly into the corresponding bromosilane. 
Table 1 Synthesis of fluorous-tagged silaketals $7 \mathbf{a}-\mathbf{l}$ (Scheme 2)

\begin{tabular}{|c|c|c|c|c|c|}
\hline Entry & Substrate & Method $^{a}$ & Product & Yield (and purity) after F-SPE only $(\%)^{b}$ & Yield of purified product $(\%)^{c}$ \\
\hline 1 & $12^{d}$ & A & ent $-7 \mathrm{~g}$ & - & Trace $^{e}$ \\
\hline $2 \mathrm{a}$ & 13a & $\mathrm{B}$ & $7 a$ & $98(65)^{f}$ & $\ldots$ \\
\hline $2 b$ & $r a c-\mathbf{1 3 d}$ & B & $r a c-7 \mathbf{d}$ & - & 56 \\
\hline $3 \mathrm{a}$ & $13 a$ & $\mathrm{C}$ & $7 \mathbf{a}$ & $94(98)$ & - \\
\hline $3 \mathrm{~b}$ & $13 b$ & $\mathrm{C}$ & $7 \mathrm{~b}$ & $>98(98)$ & - \\
\hline $3 \mathrm{c}$ & $13 c$ & $\mathrm{C}$ & $7 c$ & $>98(98)$ & - \\
\hline $3 \mathrm{~d}$ & $r a c-\mathbf{1 3 d}$ & $\mathrm{C}$ & $r a c-7 \mathbf{d}$ & - & 56 \\
\hline $3 \mathrm{e}$ & $13 e^{2}$ & $\mathrm{C}$ & $7 e$ & $55(93)$ & - \\
\hline $3 \mathrm{f}$ & $r a c-13 \mathbf{f}$ & $\mathrm{D}$ & rac-7f & $>98(76)$ & - \\
\hline $3 \mathrm{~g}$ & $13 \mathrm{~g}$ & $\mathrm{C}$ & $7 \mathrm{~g}$ & $96(85)$ & - \\
\hline $3 \mathrm{~h}$ & rac-13h & $\mathrm{C}$ & rac- $7 \mathbf{h}$ & - & 54 \\
\hline $3 i$ & $r a c-13 i$ & $\mathrm{C}$ & rac-7i & $93(67)$ & 59 \\
\hline $3 j$ & $r a c-\mathbf{1 3} \mathbf{j}$ & $\mathrm{C}$ & $r a c-7 \mathbf{j}$ & - & 32 \\
\hline $3 \mathrm{k}$ & $r a c-13 \mathbf{k}$ & $\mathrm{C}$ & $r a c-7 \mathbf{k}$ & - & 64 \\
\hline 31 & 131 & $\mathrm{C}$ & 71 & $95\left(>85^{g}\right)$ & - \\
\hline
\end{tabular}

${ }^{a}$ Methods: A: (i) 12 ( $0.5 \mathrm{M}$ ), ${ }^{\mathrm{i}} \mathrm{Pr}_{2} \mathrm{HSiCl}$ (1 eq.), $\mathrm{Et}{ }_{3} \mathrm{~N}$ (1.1 eq.), DMAP (0.1 eq.), $\mathrm{CH}_{2} \mathrm{Cl}_{2}, 0{ }^{\circ} \mathrm{C} \rightarrow \mathrm{rt}$; (ii) NBS (1 eq.); (iii) 6 ( 1 eq.), Et ${ }_{3} \mathrm{~N}$ (1.1 eq.), DMAP (0.1 eq.), $\mathrm{CH}_{2} \mathrm{Cl}_{2}, 0{ }^{\circ} \mathrm{C} \rightarrow \mathrm{rt}$; B: (i) 13 (3.5 eq., $\left.0.2 \mathrm{M}\right)$, NBS (3 eq.), $\mathrm{CH}_{2} \mathrm{Cl}_{2}, 0{ }^{\circ} \mathrm{C} \rightarrow \mathrm{rt}, 15 \mathrm{~min}$; (ii) add to 6 ( 1 eq., $\left.40 \mathrm{mM}\right), \mathrm{Et}_{3} \mathrm{~N}(15$ eq.), DMAP $\left(10 \mathrm{~mol} \%\right.$ ), $\mathrm{CH}_{2} \mathrm{Cl}_{2}, 0^{\circ} \mathrm{C} \rightarrow \mathrm{rt}$; (iii) F-SPE; C: (i) 13 (3.5 eq., $0.2 \mathrm{M}$ ), NBS (3 eq.), $\mathrm{CH}_{2} \mathrm{Cl}_{2}, 0^{\circ} \mathrm{C} \rightarrow \mathrm{rt}, 15$ min; (ii) inverse addition of 6 (1 eq., $0.25 \mathrm{M}$ ), DMAP $(50 \mathrm{~mol} \%), \mathrm{Et}_{3} \mathrm{~N}, 0{ }^{\circ} \mathrm{C} \rightarrow \mathrm{rt}$; (iii) F-SPE; D: (i) $\mathbf{1 3}(5.5$ eq., $0.2 \mathrm{M}), \mathrm{NBS}\left(5\right.$ eq.), $\mathrm{CH}_{2} \mathrm{Cl}_{2}, 0^{\circ} \mathrm{C} \rightarrow \mathrm{rt}$; (ii) inverse addition of $\mathbf{6}$ (1 eq., $0.25 \mathrm{M}$ ), DMAP $\left(50 \mathrm{~mol}^{\%}\right), \mathrm{Et}_{3} \mathrm{~N}, 0{ }^{\circ} \mathrm{C} \rightarrow \mathrm{rt}$; (iii) F-SPE. ${ }^{b}$ Yield of product after F-SPE; the purity, determined by analytical HPLC, is given in parentheses. ${ }^{c}$ Yield of product after purification on Florisil ${ }^{\mathbb{R}} .{ }^{d}$ An alcohol was used as the substrate; method A involves the synthesis of the diisopropylsilyl ether in situ. ${ }^{e}$ The fluorous-tagged alcohol 6 was recovered in $>98 \%$ yield. ${ }^{f}$ In the absence of DMAP, an $86 \%$ yield of material with $56 \%$ purity was obtained. ${ }^{g}$ Determined by $500 \mathrm{MHz}{ }^{1} \mathrm{H}$ NMR spectroscopy.

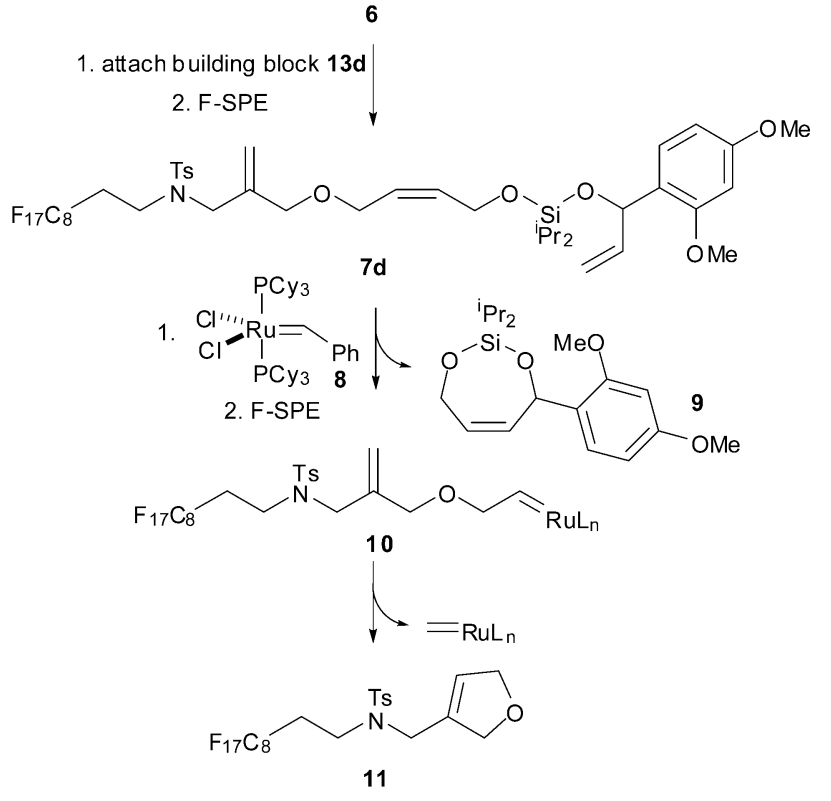

Scheme 3 Design of the fluorous-tagged linker 6 illustrated for the proposed synthesis of the cyclic silaketal 9.

With a method for the activation of a diisopropylsilyl ether in hand, we investigated the formation of unsymmetrical silaketals from the silyl ethers 13a and 13d (entries 2a-b, Table 1). The silyl ethers (3.5 eq., $0.2 \mathrm{M}$ in $\mathrm{CH}_{2} \mathrm{Cl}_{2}$ ) were treated with NBS (3 eq.) and, after $15 \mathrm{~min}$, were added to a solution of the fluoroustagged alcohol 6 (1 eq., $40 \mathrm{mM}$ ) and DMAP $(10 \mathrm{~mol} \%)$ in $\mathrm{CH}_{2} \mathrm{Cl}_{2}$. Unfortunately, neither of the reactions proceeded to completion: in each case, following F-SPE, both the fluorous-tagged alcohol 6 and the required silaketal 7 were found in the fluorous fraction. Although the silaketals could be purified by filtration through a short pad of Florisil ${ }^{\circledR}$, the method was not sufficiently efficient for application in parallel synthesis.
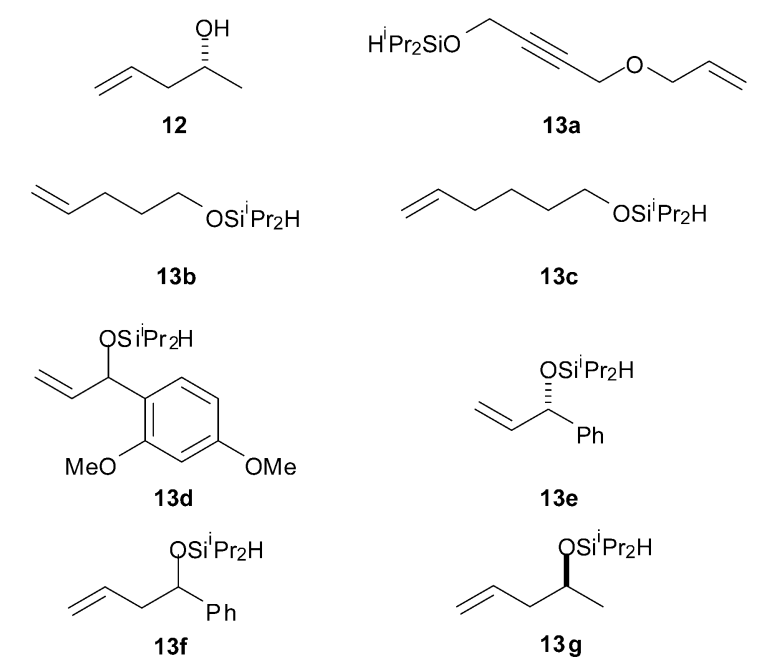

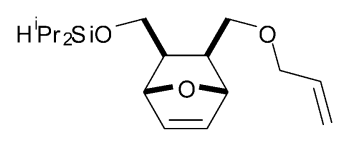

$13 h$

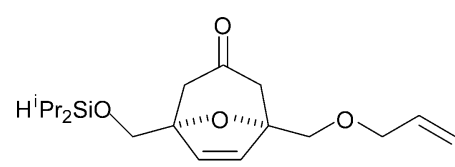

13j

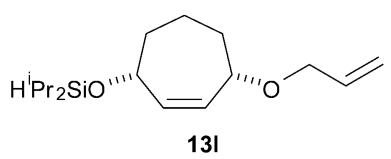

Fig. 1 Substrates, including the diisopropylsilyl ethers $\mathrm{H}^{\mathrm{i}} \mathrm{Pr}_{2} \mathrm{SiOR}(13)$, used in this study. 


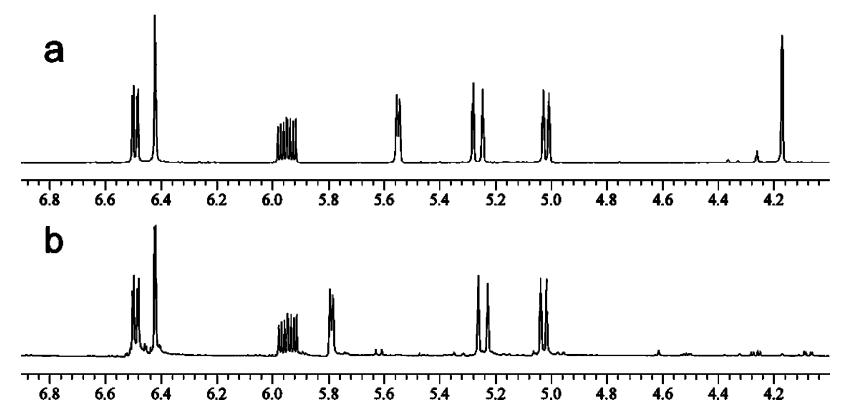

Fig. 2 Activation of the diisopropylsilyl ether $r a c-13 d .500 \mathrm{MHz}^{1} \mathrm{H}$ NMR spectra of the silyl ether 13d in $\mathrm{CDCl}_{3}$ (a) before treatment with NBS ( $\mathrm{SiH}$ : $\delta=4.2 \mathrm{ppm}$ ); and (b) $15 \mathrm{~min}$ after treatment with NBS.

We increased the efficiency of the transformation by developing a protocol in which the concentration of the fluorous-tagged alcohol 6 was higher (entries 3a-1, Table 1). The diisopropylsilyl ethers 13a-l were prepared by silylation of the corresponding alcohols, and were used without further purification. The modified procedure involved inverse addition of a solution of the fluoroustagged alcohol $\mathbf{6}$ and DMAP in triethylamine to a solution of the activated silyl ether in $\mathrm{CH}_{2} \mathrm{Cl}_{2}$. Following evaporation, addition of pentane and filtration, the crude reaction mixtures were dissolved in DMF, purified by F-SPE, and the fluorous fractions analysed, usually by HPLC. In some cases, the unsymmetrical silaketal products 7 were purified by filtration through a Florisil ${ }^{\circledR}$ pad.

The reactions of the silyl ethers 13a-c, derived from unhindered primary alcohols, were highly efficient: the unsymmetrical silaketals $7 \mathbf{a}-\mathbf{c}$ were obtained in high yield (entries $3 \mathrm{a}-\mathrm{-c}$, Table 1). With the silyl ethers $\mathbf{1 3 d}-\mathbf{g}$, derived from secondary alcohols, good yields of the corresponding silaketals $\mathbf{7 d}-\mathbf{g}$ were obtained (entries $3 \mathrm{~d}-\mathrm{f})$. We investigated more hindered bridged $(\mathbf{1 3 h}-\mathbf{j})$ and cyclic substrates (13k-l) (entries 3h-1). Even when the reaction was not highly efficient, reasonable yields of the required silaketals were obtained after filtration through a Florisi ${ }^{\circledR}$ pad.

The chemoselectivity of our method for the activation of diisopropylsilyl ethers is remarkable. The silyl ethers 13a-I contain a range of functional groups that may react with NBS: alkenes, including strained substrates $(\mathbf{1 3 h}-\mathbf{i})$; electron-rich aromatic rings (13d) and an alkyne (13a). We never observed any brominated products.

The ring-closing metatheses of five unsymmetrical fluoroustagged silaketals (7d, 7f, $7 \mathbf{h}, \mathbf{7} \mathbf{j}$ and $\mathbf{7 l}$ ) were investigated (Table 2). The silaketals 7 were treated with $3 \mathrm{~mol} \%$ portions of Grubbs' first generation catalyst $(\mathbf{8})$ until the reactions were judged to be

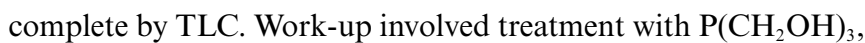
$\mathrm{Et}_{3} \mathrm{~N}$ and silica, filtration through Celite, ${ }^{10}$ and evaporation. The products of the metathesis reactions described in Table 2 are shown in Fig. 3.

Treatment of the silaketal $\mathbf{7 d}$ with the catalyst $\mathbf{8}$ triggered the release of the stable cyclic silaketal 9 from the fluorous tag (entry 1, Table 2). In the case of $\mathbf{7 h}$, however, both the expected silaketal 16 and the corresponding diol 15 were obtained after chromatography (entry $3 \mathrm{a}$ ). In later experiments, therefore, the crude cyclic silaketals were treated with HF.pyridine before purification. Using this protocol, the silaketals $7 \mathbf{f}, 7 \mathbf{h}$ and $7 \mathbf{l}$ yielded the diols 14, 15 and $\mathbf{1 8}$ (entries 2, 3b and 5).

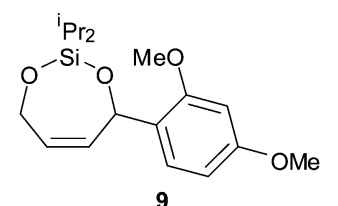<smiles>OC/C=C/CC(O)c1ccccc1</smiles><smiles>OC/C=C\C1OC2C=CCOC[C@H]2C1CO</smiles>
14

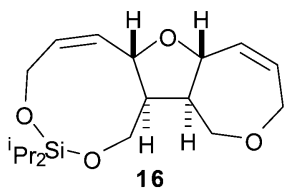<smiles>O=C1C[C@@]2(CO)C=C[C@@](COC/C=C\CO)(C1)O2</smiles><smiles>OC/C=C\C(O)CCCC1C=CCO1</smiles>

Fig. 3 Products of the ring-closing metathesis reactions.

For the substrates $\mathbf{7 d}, \mathbf{7 f}, \mathbf{7 h}$ and $\mathbf{7 l}$, the initial metathesis product was a seven- to nine-membered cyclic silaketal. Similar ring-closing metatheses are well known. ${ }^{2-4}$ However, in the case of the silaketal $7 \mathbf{j}$, the outcome of the metathesis reaction was remarkable: the diol 17 was isolated as a $55: 45 E: Z$ mixture (entry 4). The fate of $7 \mathbf{j}$ is consistent with the macrocycle-closing metathesis reaction $(\rightarrow \mathbf{2 0})$ which is described in Scheme 4. Cyclisation of 19 to form a six-membered ring was not observed: presumably six-membered ring formation is reversible, and macrocyclisation $(19 \rightarrow 20)$ allows more efficient release from the fluorous tag.

In summary, we have developed an efficient method for the formation of unsymmetrical silaketals, which we applied in the synthesis of the fluorous-tagged metathesis substrates $7 \mathbf{a}-\mathbf{l}$. The method is

Table 2 Ring-closing metathesis reactions of fluorous-tagged silaketals 7

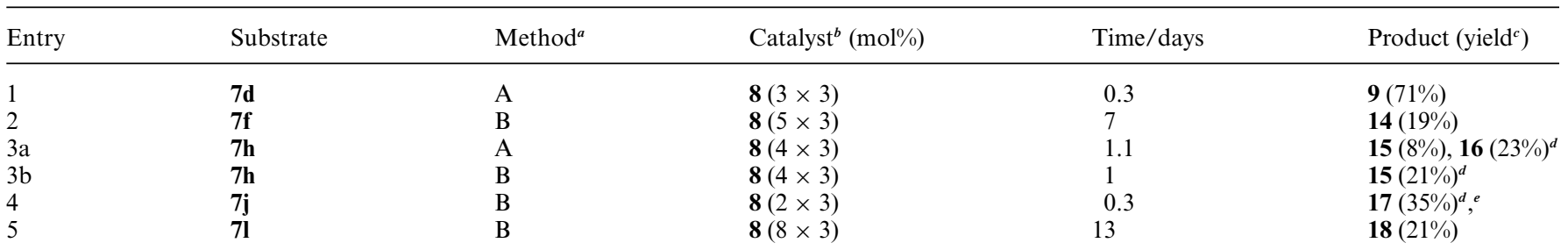

${ }^{a}$ Methods: A: (i) $7(1 \mathrm{mM}), \mathrm{CH}_{2} \mathrm{Cl}_{2}, 45^{\circ} \mathrm{C}$; (ii) $\mathrm{P}\left(\mathrm{CH}_{2} \mathrm{OH}\right)_{3}$ then $\mathrm{Et}_{3} \mathrm{~N}$; (iii) F-SPE; B: (i) $7(1 \mathrm{mM}), \mathrm{CH}_{2} \mathrm{Cl}_{2}, 45^{\circ} \mathrm{C}$; (ii) $\mathrm{P}\left(\mathrm{CH}_{2} \mathrm{OH}\right)_{3}$ then $\mathrm{Et}_{3} \mathrm{~N}$; (iii) F-SPE; (iv) HF.pyridine then $\mathrm{Me}_{3}$ SiOMe. ${ }^{b}$ The catalyst was added at regular intervals in 3 mol\% portions. ${ }^{c}$ Yield of products (see Fig. 3 ) purified by column chromatography. ${ }^{d}$ The product was purified by preparative HPLC. ${ }^{e} 55: 45 E: Z$ isomers mixture. 


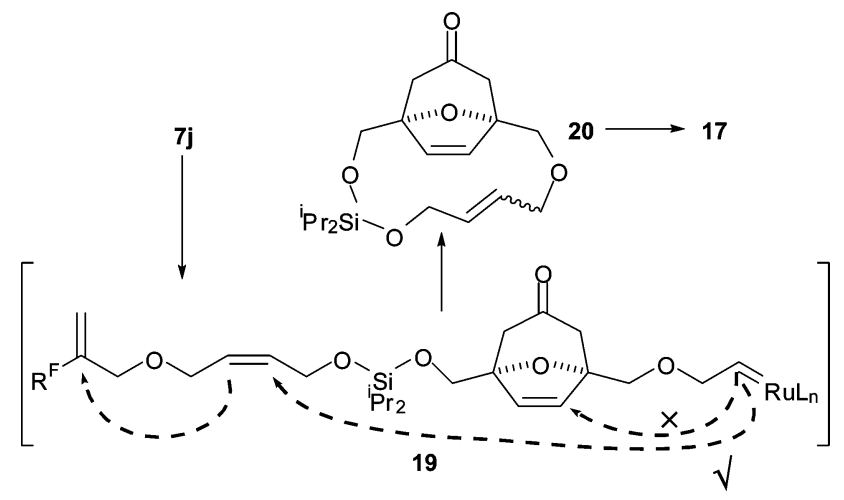

Scheme 4 Macrocycle-closing metathesis of the silaketal 7j.

remarkably chemoselective, and can be used to prepare silaketals with alkenyl and electron-rich aromatic substituents. We also observed a macrocycle-closing metathesis reaction that proceeded via a 13-membered cyclic silaketal. Through the use of a temporary silicon connection, therefore, it was possible to effect the net cross-metathesis of an unsaturated alcohol in which the reacting alkene was rather remote from the tether. Similar macrocycleclosing metathesis reactions may be useful in the synthesis of other compounds in which an alkene is remote from a potential tethering point.

\section{Acknowledgements}

We thank the Wellcome Trust, EPSRC, the University of Leeds and GSK for funding, James Titchmarsh for HPLC analyses, Simon Barrett for NMR analysis, and Dr Gordon McKiernan for helpful discussions.

\section{Notes and references}

1 M. Bols and T. Skrydstrup, Chem. Rev., 1995, 95, 1253.

2 (a) P. van de Weghe, D. Aoun, J.-G. Boiteau and J. Eustache, Org. Lett., 2002, 4, 4105; (b) P. A. Evans, J. Cui, S. J. Gharpure, A. Polosukhin and H.-R. Zhang, J. Am. Chem. Soc., 2003, 125, 14702; (c) T. Gaich and J. Mulzer, Org. Lett., 2005, 7, 1311.

3 B. A. Harrison and G. L. Verdine, Org. Lett., 2001, 3, 2157.

4 P. A. Evans, J. Cui and G. P. Buffone, Angew. Chem., Int. Ed., 2003, 42, 1734.

5 (a) C. Colombier, T. Skrydstrup and J. M. Beau, Tetrahedron Lett., 1994, 35, 8167; (b) G. Stork and P. F. Keitz, Tetrahedron Lett., 1989, 30, 6981; (c) J. W. Gillard, R. Fortin, H. E. Morton, C. Yoakim, C. A. Quesnelle, S. Daignault and Y. Guindon, J. Org. Chem., 1988, 53, 2602.

6 S. G. Leach, C. J. Cordier, D. Morton, G. J. McKiernan, S. Warriner and A. Nelson, J. Org. Chem., 2008, 73, DOI: 10.1021/jo7026273.

7 W. Zhang and D. P. Curran, Tetrahedron, 2006, 62, 11837.

8 J.-D. Moriggi, L. J. Brown, J. L. Castro and R. C. D. Brown, Org. Biomol. Chem., 2004, 2, 835.

9 M. Petit, G. Chouraqui, C. Aubert and M. Malacria, Org. Lett., 2003, 5, 2037.

10 H. D. Maynard and R. H. Grubbs, Tetrahedron Lett., 1999, 40, 4137. 